Research Paper

\title{
EMMPRIN Inhibits bFGF-Induced IL-6 Secretion in an Osteoblastic Cell Line, MC3T3-E1
}

\author{
Akari Saiki ${ }^{1}$, Mitsuru Motoyoshi ${ }^{1}$ 2, Keiko Motozawa1,3, Teinosuke Okamura ${ }^{4,5}$, Kousuke Ueki ${ }^{3,6}$, \\ Noriyoshi Shimizu ${ }^{1,2}$ and Masatake Asano $7,8 \square$
}

1. Department of Orthodontics, Nihon University School of Dentistry, 1-8-13 Kanda Surugadai, Chiyoda-ku, Tokyo 101-8310, Japan;

2. Division of Clinical Research, Dental Research Center, Nihon University School of Dentistry, 1-8-13 Kanda Surugadai, Chiyoda-ku, Tokyo 101-8310, Japan;

3. Oral Structural and Functional Biology, Nihon University Graduate School of Dentistry, 1-8-13 Kanda Surugadai, Chiyoda-ku, Tokyo 101-8310, Japan;

4. Division of Applied Oral Sciences, Nihon University Graduate School of Dentistry, 1-8-13 Kanda Surugadai, Chiyoda-ku, Tokyo 101-8310, Japan;

5. Department of Endodontics, Nihon University School of Dentistry, 1-8-13 Kanda Surugadai, Chiyoda-ku, Tokyo 101-8310, Japan;

6. Department of Oral and Maxillofacial Surgery, Division of Oral Surgery, Nihon University School of Dentistry, 1-8-13 Kanda Surugadai, Chiyoda-ku, Tokyo 101-8310, Japan;

7. Department of Pathology, Nihon University School of Dentistry, 1-8-13 Kanda Surugadai, Chiyoda-ku, Tokyo 101-8310, Japan;

8. Division of Immunology and Pathobiology, Dental Research Center, Nihon University School of Dentistry, 1-8-13 Kanda Surugadai, Chiyoda-ku, Tokyo 101-8310, Japan.

$\square$ Corresponding author: Masatake Asano, Department of Pathology, Nihon University School of Dentistry, 1-8-13 Kanda Surugadai, Chiyoda-ku, Tokyo 101-8310, Japan. Phone: +81-3-3219-8114 E-mail: asano.masatake@nihon-u.ac.jp

(c) Ivyspring International Publisher. This is an open access article distributed under the terms of the Creative Commons Attribution (CC BY-NC) license (https://creativecommons.org/licenses/by-nc/4.0/). See http://ivyspring.com/terms for full terms and conditions.

Received: 2017.04.03; Accepted: 2017.07.05; Published: 2017.09.19

\begin{abstract}
Background: Electrolytically-generated acid functional water (FW) is obtained by electrolyzing low concentrations of saline. Although it has been widely used in clinical practice with various purposes, the underlying mechanisms of action involved have not been fully elucidated so far. We used the human cervical cancer-derived fibroblastic cell line (HeLa), to examine the cytokine secretion profile following FW treatment in the present study.

Results: FW stimulation significantly induced the secretion of basic fibroblast growth factor (bFGF) and extracellular matrix metalloproteinase inducer (EMMPRIN). The effect of both factors on osteoblast-like MC3T3-El cells was further examined by stimulating the cells with the conditioned medium of FW-stimulated HeLa cells. However, the conditioned medium failed to induce IL-6 secretion. The MC3T3-El cells were further stimulated with recombinant bFGF or EMMPRIN or a combination of both factors. Intriguingly, bFGF-stimulated IL-6 induction was totally inhibited by EMMPRIN. Pretreatment with the specific inhibitor of nuclear factor-kappa $B$ (NF-KB) drastically inhibited IL-6 secretion indicating that bFGF-induced IL-6 expression was dependent on NF-KB activation. The phosphorylation status of NF-KB p65 subunit was further examined. The results indicated that EMMPRIN inhibited bFGF-induced NF-KB p65 phosphorylation.

Conclusions: These findings suggest that bFGF can induce IL-6 secretion in MC3T3-El cells through NF-KB activation. As EMMPRIN inhibited bFGF-induced IL- 6 secretion by reducing the p65 subunit phosphorylation, it might be concluded that bFGF and EMMPRIN crosstalk in their respective signaling pathways.
\end{abstract}

Key words: EMMPRIN, bFGF, IL-6, osteoblast.

\section{Introduction}

The periodontium is composed of four different tissues, gingiva, cementum, periodontal ligament and alveolar bone, all of which support the teeth in the oral cavity. In this context, stratified squamous epithelial cells (SSEC), fibroblasts (the main component of the gingiva), and both osteoblasts as well as osteoclasts lie in close proximity to each other within the periodontium. These cells cross talk via 
cytokines and chemokines and establish the functional relationship required to maintain oral homeostasis.

Electrolytically-generated acid functional water (FW) is obtained by electrolyzing low concentrations saline, and is widely used as a disinfectant in clinical practice [1-3]. In a previous report, FW was demonstrated to induce the expression of human $\beta$-defensin $2(h B D 2)$ in oral squamous cell carcinoma cell lines (OSCC) [4]. Defensins are cationic, cystein-rich peptides with molecular masses ranging from 3 to $5 \mathrm{kDa}$ [5]. They function as antimicrobial components of the innate immune system. The induction of hBD-2 mRNA expression in response to various stimuli has been reported in several studies $[6,7]$; therefore, we speculated that FW-mediated $h B D 2$ expression could be induced at the transcriptional level.

SSEC are absent on the surface of the oral cavity during injury as a result of which, the fibroblasts are directly exposed to the oral cavity. FW has been shown to accelerate the wound healing process in a burn wound model [1]. Although this effect is attributed to the disinfectant activity of FW, the underlying mechanisms involved have not been fully elucidated so far. Hence, in order to evaluate the effect of FW on fibroblasts experimentally, we used the human cervical cancer-derived fibroblastic cell line (HeLa), to examine the cytokine secretion profile following FW treatment in the present study. Augmented secretion of basic fibroblast growth factor (bFGF) and extracellular matrix metalloproteinase inducer (EMMPRIN) was detected in the cells. bFGF is a pleiotropic cytokine with a variety of functions [8]. It was found to be expressed in all stages of fracture repair [9]. EMMPRIN is a transmembrane protein and belongs to the immunoglobulin superfamily [10]. Although the major function of this protein involves the induction of matrix metalloproteinases (MMPs), it also contributes to various other biological responses [10]. In the present study, we attempted to elucidate the biological functions of FW using the widely used human fibroblastic cell line HeLa and murine osteoblastic cell line MC3T3-E1 cells and discovered the occurrence of overlapping signaling between bFGF and EMMPRIN.

\section{Methods}

\section{Reagents}

FW was kindly provided by Miura Densi (Akita, Japan). Recombinant bFGF and EMMPRIN were purchased from R\&D systems (Tokyo, Japan). L-1-4'-tosylamino-phenylethyl-chloromethyl ketone (TPCK) and mitogen-activated protein kinase (MEK) inhibitor U0126 were purchased from Sigma-Aldrich (Tokyo, Japan) and Promega (Tokyo, Japan), respectively.

\section{Cell culture and FW stimulation}

Human HeLa cells and the mouse MC3T3-E1 cells (osteoblastic cell line) were obtained from the Health Science Research Resources Bank (Osaka, Japan) and Riken (Ibaraki, Japan), respectively. Each cell line was maintained in a-minimum essential medium (a-MEM) or Dulbecco's modified eagle medium (DMEM) supplemented with 10\% FCS, 50 $\mathrm{mg} / \mathrm{ml}$ streptomycin, and $50 \mathrm{U} / \mathrm{ml}$ penicillin $(10 \%$ FCS-a-MEM or $10 \%$ FCS-DMEM). The MC3T3-E1 cells were plated on a 6-well plate (IWAKI, Tokyo, Japan) at a density of $5 \times 10^{4}$ /well in $2 \mathrm{ml}$ of $10 \%$ FCS-a-MEM. After 5 days, the medium was replaced with $2 \mathrm{ml}$ of a-MEM containing 0.3\% FCS. The cells were used for experiment after $48 \mathrm{~h}$.

\section{Cytokine array experiment}

HeLa cells were plated on a $10 \mathrm{~cm}$ cell culture dish (Greiner, Tokyo, Japan) at a density of $1 \times 10^{6}$ /well the day before the experiment. Following stimulation with FW for $30 \mathrm{sec}$, the cells were washed and further cultured for $6 \mathrm{~h}$. The culture supernatants were collected and subjected to cytokine array experiments (R\&D systems, Tokyo, Japan) according to the manufacture's protocol. Images were taken using ChemiDoc XRS (BioRad, Tokyo, Japan).

\section{Real-time PCR}

Total RNA was purified using the RNeasy mini kit (QIAGEN, Tokyo, Japan). Complementary DNA (cDNA) was synthesized using Superscript III reverse transcriptase (Invitrogen, San Diego, CA, USA) and subjected to real-time PCR, as described previously [11]. Real-time PCR was performed using the CFX96-Real-Time-System (BioRad, Tokyo, Japan) with SYBR green (TaKaRa, Tokyo, Japan). The primers used in this study are listed in Table 1.

Table 1. The primers used in this study.

\begin{tabular}{llll}
\hline Target Gene & & Oligonucleotide Sequence & Genbank acc. No. \\
\hline$\beta$-actin & Forward primer & 5'-GGAGCAAGTATCTTGATCTTC-3' & NM_007393 \\
& Reverse primer & 5'-CCTTCCTGCGCATGGAGTCCTG-3' & \\
IL-6 & Forward primer & 5'-CCACTTCACAAGTCGGAGGCTTA-3' & NM_031168.1 \\
& Reverse primer & 5'-CCAGTTTGGTAGCATCCATCATTTC-3' & \\
\hline
\end{tabular}




\section{Enzyme-linked immunosorbent assay (ELISA)}

HeLa cells $\left(5 \times 10^{5}\right)$ were plated on a 6 -well plate and stimulated with FW for $30 \mathrm{sec}$. The cells were further cultured for 1,3 and $6 \mathrm{~h}$. The culture supernatants and cell lysates $(1 \mathrm{ml})$ were harvested, and concentrations of bFGF and EMMPRIN were measured using the DuoSet ELISA Development System (R\&D Systems, Tokyo, Japan). For interleulkin-6 (IL-6) measurements, MC3T3-E1 cells were stimulated with one of the following: FW-stimulated HeLa cell-derived conditioned medium; recombinant human (rh) bFGF (at concentrations of $0,0.01,0.1,1,3$, and $10 \mathrm{nM}$ ); rh EMMPRIN $(0,0.5,1$, and $2 \mu \mathrm{g} / \mathrm{ml})$; or both rh bFGF and rh EMMPRIN for $1 \mathrm{~h}$. IL-6 and vascular endothelial growth factor (VEGF) concentrations were measured with IL-6 and VEGF ELISA kits (R\&D systems). For the inhibitor experiments, the cells were pre-treated with TPCK $(1,10,25 \mu l)$ or MEK inhibitor $\mathrm{U} 0126(1,10,100 \mu \mathrm{l})$ for $1 \mathrm{~h}$.

\section{Western blotting}

MC3T3-E1 cells were stimulated with rh bFGF, rh EMMPRIN or rh bFGF and rh EMMPRIN for 20 min. The cells were then washed twice with ice cold PBS and lysed with $100 \mu \mathrm{l}$ of lysis buffer $(50 \mathrm{mM}$ Tris- $\mathrm{HCl}, \mathrm{pH} 7.5,150 \mathrm{mM} \mathrm{NaCl}$, and $0.5 \%$ Triton $\mathrm{X}-100)$. Protein concentrations were measured using the BioRad protein assay kit (BioRad), and $20 \mu \mathrm{g}$ of total protein was subjected to $12 \%$ SDS-PAGE. Western blotting was performed as described previously [11]. Primary antibodies (Abs) against nuclear factor-kappa B (NF-kB) p65 subunit $(\times 200)$, phosphorylated p65 subunit $(\times 200)$ and GAPDH $(\times$ $10,000)$ were diluted with $1 \%$ BSA-PBST $(0.1 \%$ tween-20/PBS). Anti-p38 and anti-p-p38 Abs were diluted to $\times 500$, whereas the secondary goat anti-mouse $\operatorname{IgG}(\mathrm{H}+\mathrm{L})$ and goat anti-rabbit $\operatorname{IgG}(\mathrm{H}+\mathrm{L})$ Abs were diluted to $\times 10,000$ using $1 \%$ BSA-PBST. The bands were detected using the ECL kit (GE Healthcare, Tokyo, Japan).

\section{Statistical analysis}

The one-way ANOVA with Tukey-Kramer tests was used for all statistical analyses. Results are presented as mean \pm standard deviation (SD). P values $<0.05$ were considered as statistically significant.

\section{Results}

\section{FW induced bFGF and EMMPRIN secretion}

FW stimulation significantly augmented endoglin, bFGF and EMMPRIN secretion in the cells; on the other hand, PDGF-AA and VEGF were down regulated by FW (Fig. 1a). To confirm these results, concentrations of each factor were measured by ELISA. Culture supernatants and cell lysates of the FW-stimulated cells were collected at 1,3 and $6 \mathrm{~h}$. The concentration of bFGF reached $180 \mathrm{pg} / \mathrm{ml}$ after $1 \mathrm{~h}$ and was found to be maintained even at $6 \mathrm{~h}$ after stimulation (Fig. 2a). Conversely, in the cell lysates, bFGF concentrations were found to be slightly lowered, although the decrease was not statistically significant. EMMPRIN secretion increased in a time-dependent manner and reached up to $1.8 \mathrm{ng} / \mathrm{ml}$ and $4.5 \mathrm{ng} / \mathrm{ml}$ after 1 and $6 \mathrm{~h}$ of stimulation, respectively (Fig. 2b). A gradual decrease in the concentration of EMMPRIN was noted in the cell lysates, and reached statistical significance after $6 \mathrm{~h}$. In contrast, apparent decrease in endoglin levels was observed in the supernatants and cell lysates (Fig. 2c).

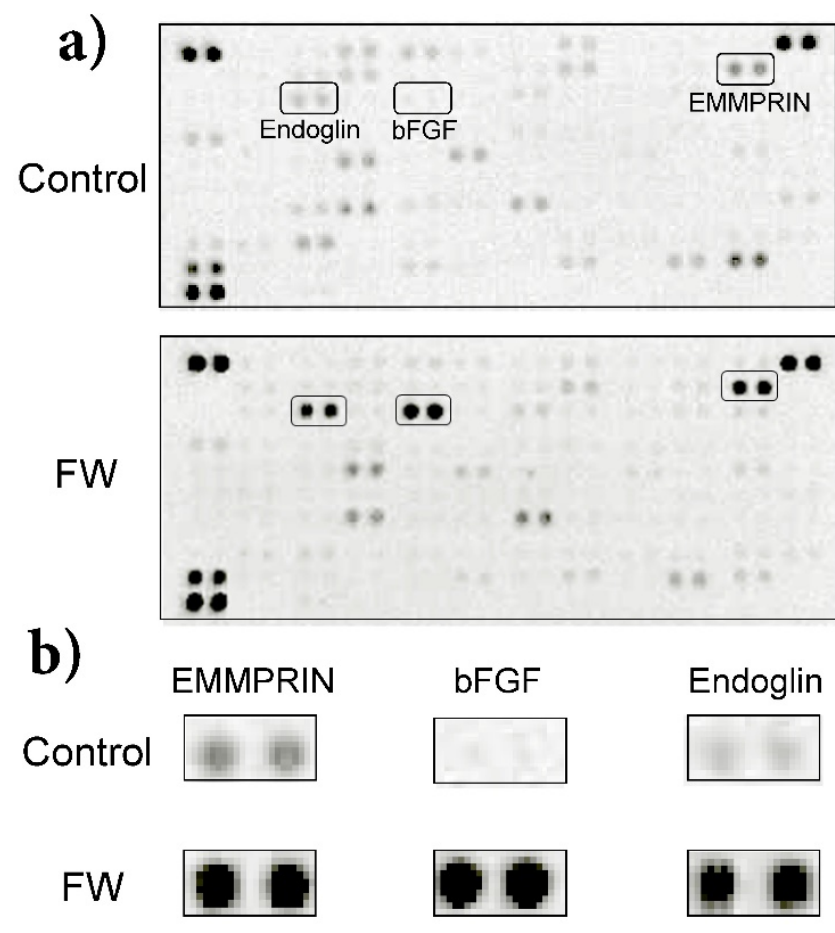

Figure 1 a) HeLa cells $\left(1 \times 10^{6}\right)$ were stimulated with (lower panel) or without FW (upper panel: controls) for $30 \mathrm{sec}$. The stimulation was stopped with $10 \%$ FCS-DMEM. The cells were washed and cultured with fresh $10 \%$ FCS-DMEM for $6 \mathrm{~h}$. Culture supernatants were harvested and subjected to a cytokine array experiment. b) Enlarged views of the boxed areas in (a).

\section{Effect of bFGF and EMMPRIN on osteoblasts}

MC3T3-E1 cells were cultured with FW-stimulated or non-stimulated HeLa cell-derived conditioned medium. As bFGF is demonstrated to induce the production of IL-6 and VEGF in MC3T3-E1 cell $[12,13]$, we examined the production of these factors. Unexpectedly, no augmentation in the production of IL-6 or VEGF was observed in the supernatants (Fig. 3a). In order to rule out the possibility of the MC3T3-E1 cells being in a refractory 
state, they were cultured with or without recombinant bFGF or EMMPRIN. The rh bFGF and rh EMMPRIN were used for the experiments because the effectiveness of these molecules on murine cell lines have been proven [12-14]. As shown in Figure 3b, rh bFGF induced the production of both factors with peak induction observed at $3 \mathrm{nM}$ for both IL-6 and VEGF (Fig. 3b). On the contrary, rh EMMPRIN did not induce the secretion of either of the two factors (Fig. 3c).

\section{The crosstalk between bFGF and EMMPRIN}

We speculated that the lack of IL-6 induction by HeLa-derived conditioned medium might be attributed to the cross talk between bFGF and EMMPRIN in their signaling pathways. To test this possibility, MC3T3-E1 cells were cultured with rh
bFGF, rh EMMPRIN or a combination of both, and the concentration of IL-6 in the culture supernatants was measured. Consistent with previous results, rh bFGF, but not rh EMMPRIN, induced IL-6 secretion in the cells. However, rh bFGF failed to induce IL-6 secretion in the presence of rh EMMPRIN (Fig. 4a). To examine whether the inhibitory effect of rh EMMPRIN on IL-6 induction occurs at the transcriptional level, real-time PCR was performed. The cells stimulated with rh bFGF (3 nM) alone induced a 6.5-fold expression of IL-6 mRNA (Fig. 4b), whereas rh EMMPRIN did not exhibit any influence on IL-6 expression. On the other hand, rh bFGF-induced IL-6 mRNA expression was drastically reduced by rh EMMPRIN (Fig. 4b), indicating the possibility of cross talks between bFGF and EMMPRIN.
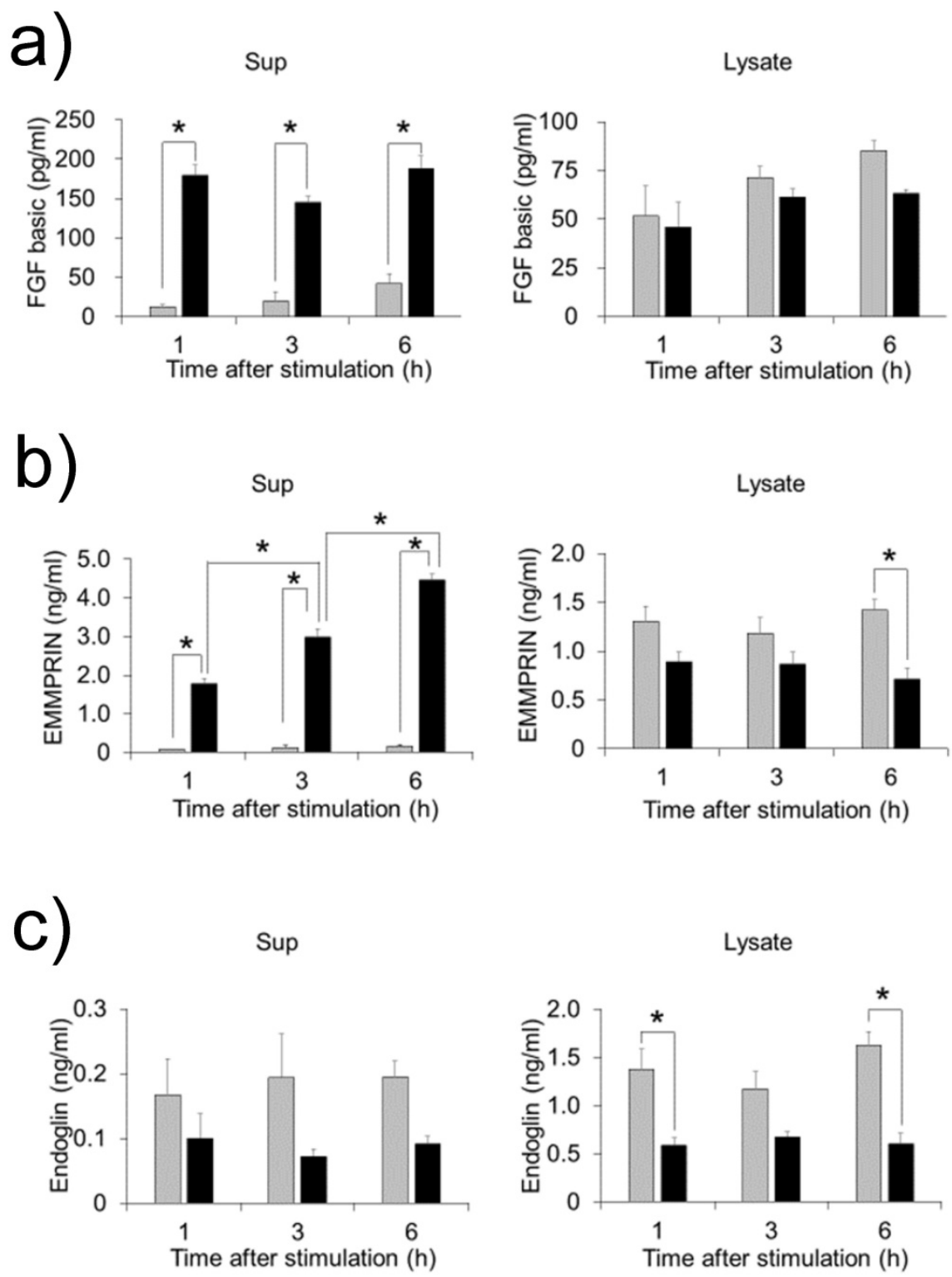

Figure 2. HeLa cells were stimulated with FW and cultured for 1, 3 and $6 \mathrm{~h}$. The culture supernatants (left) and cell lysates (right) were collected and subjected to ELISA. The mean of 4 separate experiments are shown. ${ }^{*} \mathrm{p}<0.05$ 

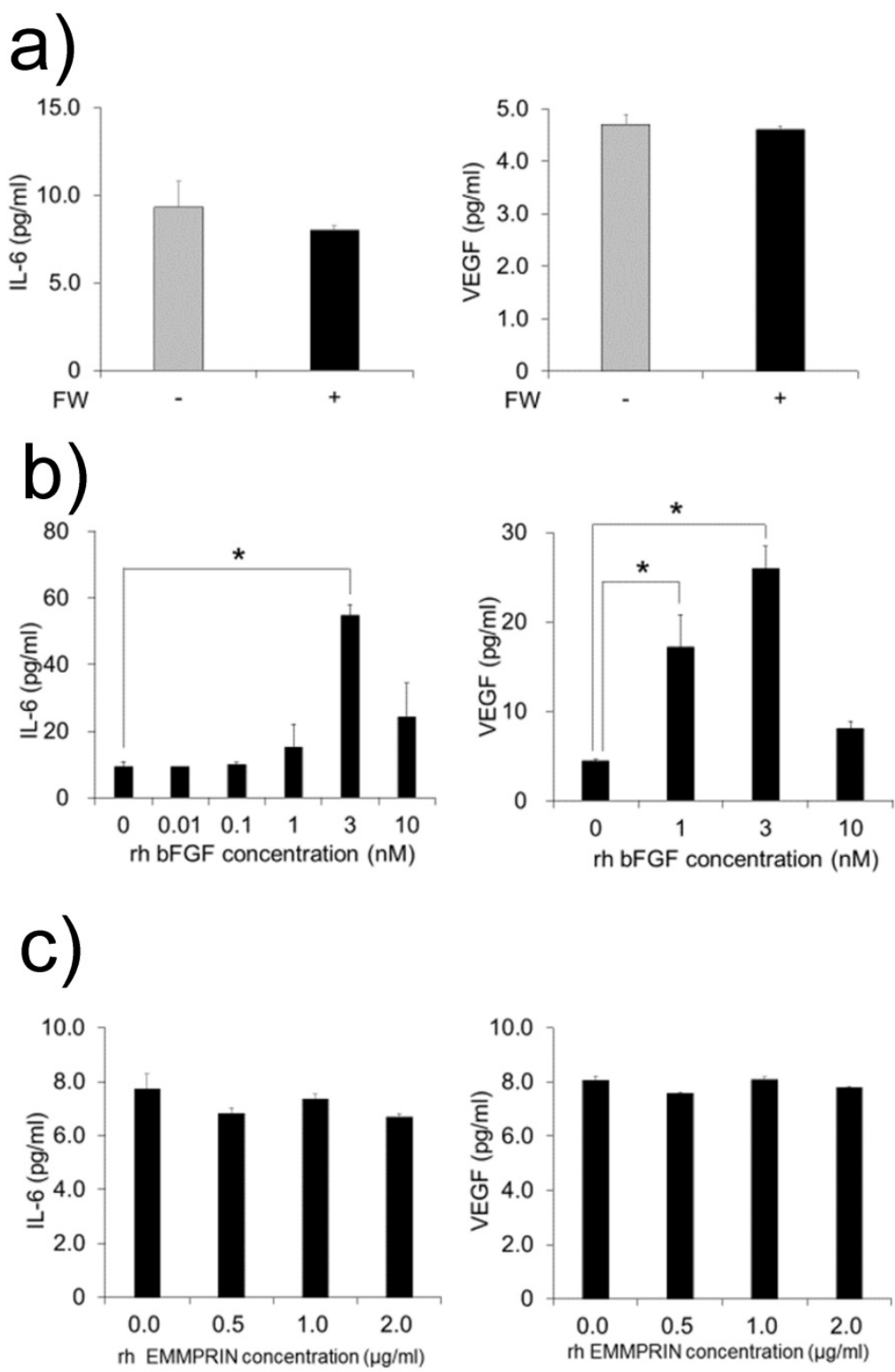

Figure 3. a) MC3T3-El cells were cultured with conditioned medium derived from FW-stimulated or -unstimulated HeLa cells for $1 \mathrm{~h}$ and further cultured with fresh $10 \%$ FCS- $\alpha M E M$ for $24 \mathrm{~h}$. The culture supernatants were harvested and subjected to IL-6 (left) and VEGF (right) ELISA. The MC3T3-El cells were cultured with b) rh bFGF at concentrations of $0,0.01,0.1,1,3$ and $10 \mathrm{nM}$ (IL-6, left) or $0,1,3$ and $10 \mathrm{nM}$ (VEGF, right) for $1 \mathrm{~h}$. The cells were cultured with c) EMMPRIN at concentrations of $0,0.5,1$ and $2 \mu \mathrm{g} / \mathrm{ml}$ (IL-6, left) or $0,0.5,1$ and $2 \mu \mathrm{g} / \mathrm{ml}$ (VEGF, right) for $1 \mathrm{~h}$. The medium was exchanged after $1 \mathrm{~h}$ of stimulation, and the cells were incubated for $24 \mathrm{~h}$. Culture supernatants were harvested and subjected to IL-6 ELISA. The mean of at least four independent experiments are shown. * $p<0.05$

\section{The signaling pathway}

To examine the signaling pathway downstream of bFGF, the cells were pre-treated with specific inhibitors against NF-KB (TPCK) and MEK (U0126). A major inhibitory effect was observed for TPCK at a concentration of $1 \mu \mathrm{M}$ (Fig. 5a). However, the MEK inhibitor did not demonstrate any inhibitory effect on IL-6 secretion at concentrations of 1 or $10 \mu \mathrm{M}$ (Fig. 5a).

These findings indicated the contribution of NF- $\mathrm{kB}$ on rh bFGF-induced IL-6 secretion in MC3T3-E1 cells. Therefore, based on these observations, we examined the influence of $\mathrm{rh}$ EMMPRIN on NF-kB signaling. The cells were stimulated with or without rh bFGF or rh EMMPRIN after which, the p65 phosphorylation status was examined by Western blotting. Phosphorylated p65 was not detected in the rh EMMPRIN-treated or control cells. On the other hand, p-p65 was clearly observed in the cells stimulated by rh bFGF. In the presence of rh EMMPRIN, however, rh bFGF-induced p-p65 was drastically reduced. As bFGF-induced IL-6 secretion is dependent on p38 phosphorylation, we examined the p38 phosphorylation status in the cells; no augmentation in p38 phosphorylation was noted irrespective of the presence of $\mathrm{rh}$ bFGF or $\mathrm{rh}$ EMMPRIN (Fig. 5c). 
a)

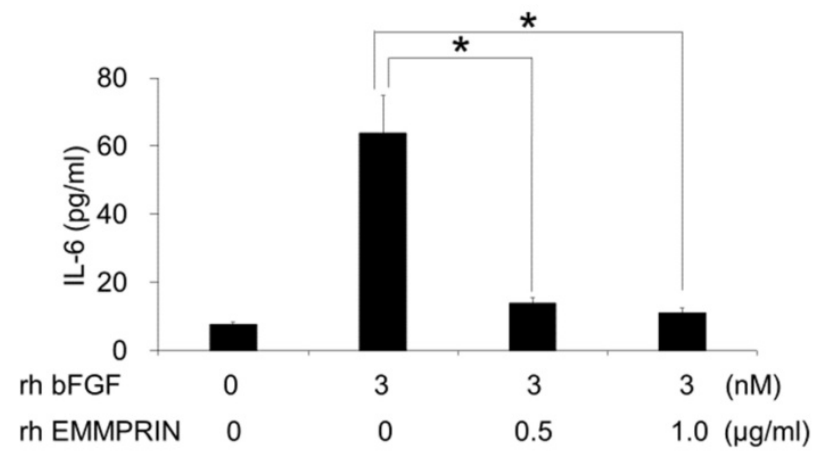

b)

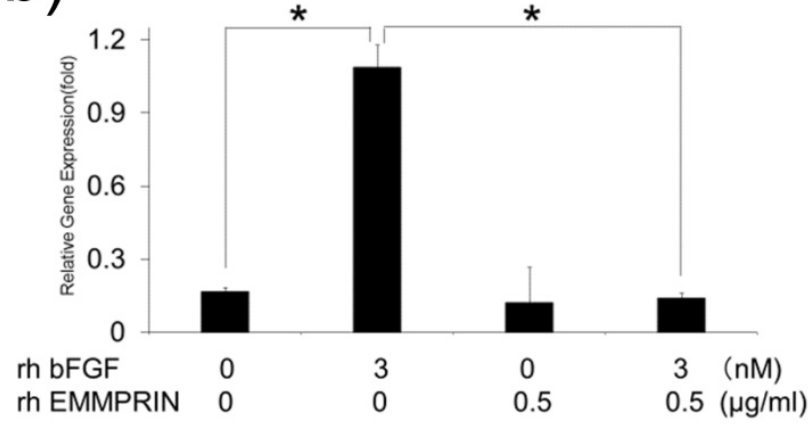

Figure 4. a) MC3T3-El cells were cultured with rh bFGF, rh EMMPRIN or a combination of both for $1 \mathrm{~h}$. The medium was exchanged after $1 \mathrm{~h}$ of stimulation, and the cells were incubated for 24 h. IL- 6 concentrations in the culture supernatants were measured with IL-6 ELISA. b) MC3T3-EI cells were cultured as in a) for $20 \mathrm{~min}$. The medium was exchanged after $20 \mathrm{~min}$ of stimulation, and cells were incubated for $3 \mathrm{~h}$. Total RNA was purified, following which, complementary DNA was generated and subjected to real-time PCR. The mean of at least four different experiments are shown. ${ }^{*} \mathrm{p}<0.05$

\section{Discussion}

In the present study, we have shown that bFGF and EMMPRIN are released from HeLa cells in response to $\mathrm{FW}$ stimulation. As $\mathrm{FW}$ has a low $\mathrm{pH}$ value, the HeLa cells are expected to be damaged to some extent. A diverse set of molecules, referred to as alarmins, are released from damaged cells in response to infection or injury, and play important roles in evoking both innate and adaptive immunity [15]. Alarmins are composed of various factors including as defensins, cathelicidin, high mobility group box protein 1 (HMGB1), interleulkin (IL)-1a, and IL-33[15]. In addition, alarmins are rapidly released in response to injury, are chemotactic to antigen presenting cells, and possess immunoenhancing effects. Intriguingly, IL-1a and bFGF share certain common features: both molecules have high molecular weight (HMW) isoforms (immature IL-1a and HMW bFGF). The N-terminal regions of both isoforms contain nuclear localizing signals (NLS) that contribute to the transcriptional control of the target genes. Moreover, these molecules do not follow the classical endoplasmic reticulum (ER)-Golgidependent secretory machinery due to the lack of signal peptides [16, 17]. Thus, the possibility of both bFGF and EMMPRIN being novel alarmins is presently being investigated in our laboratory.

bFGF is a member of the FGF family and has pleiotropic effects on various biological reactions. bFGF contributes to the wound healing by inducing new blood vessel formation. Increased bFGF expression has been observed in glial cells during brain hypothermia [18]. Moreover, induced bFGF protects neuronal apoptosis [19] and contributes to wound healing in the brain [20]. In this context, bFGF released from fibroblasts might protect the surrounding tissue from the further damage or apoptosis. Accelerated wound healing observed in the burn model [1] might be attributed to this beneficial effect of FW. On the other hand, EMMPRIN belongs to the immunoglobulin superfamily and plays important roles in several developmental processes as well as pathological conditions [10]. The main function of EMMPRIN is to induce the expression of MMPs, which belong to the zinc-dependent family of endopeptidases. MMPs play an intrinsic role in extracellular matrix remodeling [21], which is an important step during the process of wound healing. The possible induction of MMPs in MC3T3-E1 cells by FW-induced EMMPRIN is currently under investigation.

IL-6 is an important factor for bone metabolism [22]. Previous reports have demonstrated bFGF-mediated IL-6 induction in MC3T3-E1 cells [12, 23]. Based on these reports, we attempted to examine the effect of bFGF on MC3T3-E1 cells. The binding of bFGF to structurally related receptors (FGFR 1-4) activates protein kinase $\mathrm{C}$ and phospholipase $\mathrm{C}$, and culminates in p38 phosphorylation as well as IL-6 secretion [12]. In the present study, bFGF consistently induced IL-6 secretion in the cells; however, phosphorylation of p38 was not regularly observed. The results of Western blotting suggest the occurrence of constitutive low level p38 phosphorylation, which might have an effect on the IL-6 induction machinery. However, further studies investigating the precise mechanisms involved are merited. Nevertheless, instead of p38, bFGF-induced IL-6 secretion was drastically inhibited by NF-kB inhibitor in the present study. This observation is in line with a previous report indicating the importance of NF-KB for IL-6 induction [24]. As NF-KB p65 subunit is not a direct substrate of MAPK [24], the reason for the constitutive low-level p38 phosphorylation in this study needs to be examined in future. 


\section{a)}
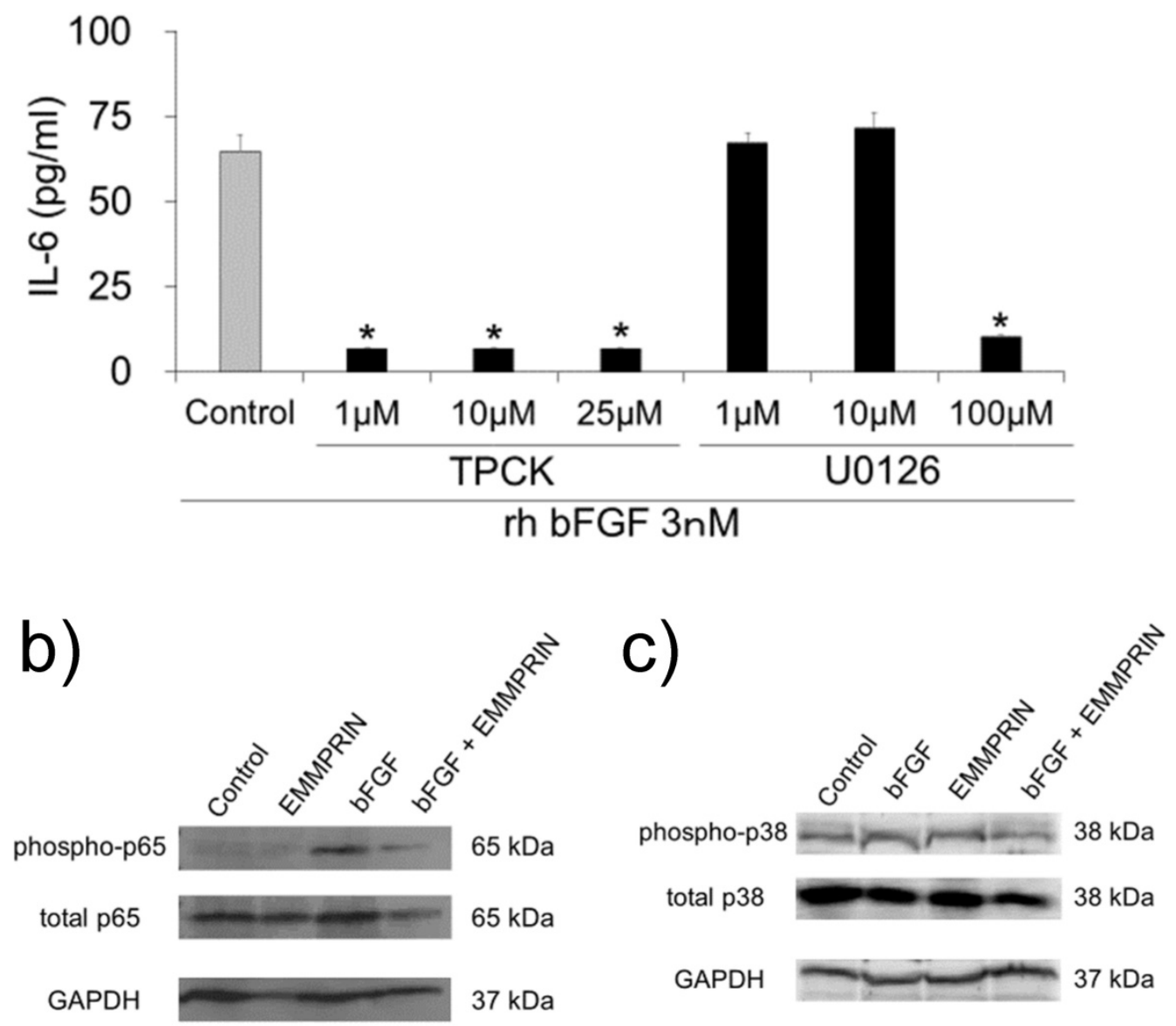

Figure 5. a) MC3T3-E1 cells were pre-incubated with 1,10 and $25 \mu \mathrm{M}$ of TPCK or 1,10 and $100 \mu \mathrm{M}$ of MEK inhibitor for 1 h. The cells were washed and further cultured with $3 \mathrm{nM}$ of rh bFGF for $1 \mathrm{~h}$. The medium was exchanged after $1 \mathrm{~h}$ of stimulation, and the cells were incubated for $24 \mathrm{~h}$. The culture supernatants were subjected to IL-6 ELISA. After stimulation with rh bFGF, rh EMMPRIN or the combination of both for 20 min, the cell lysates were harvested and subjected to Western blot b) phospho-p65 (upper), total p65 (middle) or GAPDH (lower); and c) phospho-p38 (upper), total p38 (middle) and GAPDH (lower). The representative of three independent experiments are shown.

The bFGF-mediated induction of IL-6 is significantly inhibited by EMMPRIN. EMMPRIN can interact with various molecules including EMMPRIN itself [10]. The binding of this molecule with its natural ligand cyclophilin A (CyPA) induces the activation of extracellular signal-regulated kinase (ERK; [25, 26]). In pancreatic cancer cells, CyPA promotes colocalization of EMMPRIN with CD44, and phosphorylates the signal transducer and activator of transcription 3 (STAT3; [27]). The inhibitory effect of EMMPRIN on bFGF-induced IL-6 secretion might be due to the cross talk between the NF-KB and STAT signaling pathways.

In the present study, we have demonstrated that bFGF-mediated IL-6 secretion was significantly inhibited by EMMPRIN. The inhibition of bFGF-induced NF-kB activation by EMMPRIN indicates the presence of cross-talk between the signaling pathways.

\section{Conclusions}

The bFGF induced IL-6 secretion in MC3T3-E1 cells was inhibited by EMMPRIN through reduction of NF-kB p65 subunit phosphorylation. These findings suggest the crosstalk between bFGF and EMMPRIN in their signaling pathways.

\section{Abbreviations}

FW: Electrolytically-generated acid functional water, HeLa: human cervical cancer-derived fibroblastic cell line, bFGF: basic fibroblast growth factor, EMMPRIN: extracellular matrix metalloproteinase inducer, IL-6: interleukin-6, VEGF: vascular endothelial growth factor, NF-KB: nuclear factor-kappa B, SSEC: stratified squamous epithelial cell, hBD2: human $\beta$-defensin 2, OSCC: oral squamous cell carcinoma cell line, MMPs: matrix metalloproteinases, MEK: mitogen-activated protein kinase, a-MEM: a-minimum essential medium, 
DMEM: Dulbecco's modified Eagle's medium, cDNA: complementary DNA, ELISA: enzyme-linked immunosorbent assay, rh: recombinant human, Abs: antibodies, SD: standard deviation, HMGB1: high mobility group box protein 1, IL: interleukin, HMW: high molecular weight, NLS: nuclear localizing signals, ER: endoplasmic reticulum, CyPA: cyclophilin A, ERK: extracellular signal-regulated kinase, STAT3: signal transducer and activator of transcription 3

\section{Acknowledgement}

This study was supported in part by research grants from Sato Fund from the Nihon University School of Dentistry and a grant from the Dental Research Center, Nihon University School of Dentistry, a Nihon University multidisciplinary research grant and Individual Research Grant and MEXT-Supported Program for the Strategic Research Foundation at Private Universities 2013-2017, Grant-in-Aid for Scientific Research (C) (MEXT).

\section{Competing Interests}

The authors have declared that no competing interest exists.

\section{References}

1. Nakae H, Inaba H. Electrolyzed strong acid aqueous solution irrigation promotes wound healing in a burn wound model. Artif Organs. 2000; 24: 544-546.

2. Kubota A, Goda T, Tsuru T, Yonekura T, Yagi M, Kawahara H, Yoneda A, Tazuke Y, Tani G, Ishii T, Umeda S, Hirano K. Efficacy and safety of strong acid electrolyzed water for peritoneal lavage to prevent surgical site infection in patients with perforated appendicitis. Surg Today. 2015; 45: 876-879.

3. Cheng X, Tian Y, Zhao C, Qu T, Ma C, Liu X, Yu Q. Bactericidal effect of strong acid electrolyzed water against flow Enterococcus faecalis biofilms. J Endod. 2016; 42: 1120-1125.

4. Gojoubori T, Nishio Y, Asano M, Nishida T, Komiyama K, Ito K. Distinct signaling pathways leading to the induction of human beta-defensin 2 by stimulating an electrolyticaly-generated acid functional water and double strand RNA in oral epithelial cells. J Recept Signal Transduct Res. 2014; 34: 97-103.

5. Semple CA, Gautier P, Taylor K, Dorin JR. The changing of the guard: Molecular diversity and rapid evolution of $\beta$-defensins. Mol Divers. 2006; 10 : 575-584

6. Liu L, Wang I, Jia HP, Zhao C, Heng HH, Schutte BC, McCray PB, Ganz T. Structure and mapping of the human $\beta$-defensin HBD-2 gene and its expression at sites of inflammation. Gene. 1998; 222: 237-244.

7. Krisanaprakornkit S, Kimball JR, Weinberg A, Darveau RP, Bainbridge BW, Dale BA. Inducible expression of human $\beta$-defensin 2 by Fusobacterium nucleatum in oral epithelial cells: multiple signaling pathways and role of commensal bacteria in innate immunity and the epithelial barrier. Infect Immun. 2000; 68: 2907-2915.

8. Bikfalvi A, Klein S, Pintucci G, Rifkin DB. Biological roles of fibroblast growth factor-2. Endocr Rev. 1997; 18: 26-45.

9. Bolander ME. Regulation of fracture repair by growth factors. Proc Soc Exp Biol Med. 1992; 200: 165-170.

10. Iacono KT, Brown AL, Greene MI, Saouaf SJ. CD147 immunoglobulin superfamily receptor function and role in pathology. Exp Mol Pathol. 2007; 83: 283-295.

11. Omagari D, Mikami Y, Suguro H, Sunagawa K, Asano M, Sanuki E, Moro I, Komiyama K. Poly I: C-induced expression of intercellular adhesion molecule-1 in intestinal epithelial cells. Clin Exp Immunol. 2009; 156: 294-302.

12. Kozawa $\mathrm{O}$, Tokuda $\mathrm{H}$, Matsuno $\mathrm{H}$, Uematsu $\mathrm{T}$. Involvement of p38 mitogen-activated protein kinase in basic fibroblast growth factor-induced interleukin-6 synthesis in osteoblasts. J Cell Biochem. 1999; 74: 479-485.

13. Tokuda $\mathrm{H}$, Kozawa $\mathrm{O}$, Uematsu T. Basic fibroblast growth factor stimulates vascular endothelial growth factor release in osteoblasts: divergent regulation by $\mathrm{p} 42 / \mathrm{p} 44$ mitogen-activated protein kinase and p38 mitogen-activated protein kinase. J Bone Miner Res. 2000; 15: 2371-2379.
14. Chen L, Nakai M, Belton RJ Jr, Nowak RA. Expression of extracellular matrix metalloproteinase inducer and matrix metalloproteinases during mouse embryonic development. Reproduction. 2007; 133: 405-414.

15. Oppenheim JJ, Yang D. Alarmins: chemotactic activators of immune responses. Curr Opin Immunol. 2005; 17: 359-365.

16. Dinarello C. A. Biologic basis for interleukin-1 in disease. Blood. 1996; 87: 2095-2147.

17. Delrieu I. The high molecular weight isoforms of basic fibroblast growth factor (FGF-2): an insight into an intracrine mechanism. FEBS Lett. 2000; 468: 6-10.

18. Wang Q, Ishikawa T, Michiue T, Zhu BL, Guan DW, Maeda H. Evaluation of human brain damage in fatalities due to extreme environmental temperature by quantification of basic fibroblast growth factor (bFGF), glial fibrillary acidic protein (GFAP), S100b and single-stranded DNA (ssDNA) immunoreactivities. Forensic Sci Int. 2012; 219: 259-264.

19. Alzheimer C, Werner S. Fibroblast growth factors and neuroprotection. Adv Exp Med Biol. 2002; 513: 335-351.

20. Do Carmo Cunha J, de Freitas Azevedo Levy B, de Luca BA, de Andrade MS, Gomide VC, Chadi G. Responses of reactive astrocytes containing S100beta protein and fibroblast growth factor- 2 in the border and in the adjacent preserved tissue after a contusion injury of the spinal cord in rats: implications for wound repair and neuroregeneration. Wound Repair Regen. 2007; 15: 134-146.

21. Kessenbrock K, Plaks V, Werb Z. Matrix metalloproteinases: regulators of the tumor microenvironment. Cell. 2010; 141: 52-67.

22. Roodman G. D. Perspectives interleukin-6: an osteotropic factor? J Bone Miner Res. 1992; 7: 475-478.

23. Kozawa O, Suzuki A, Uematsu T. Basic fibroblast growth factor induces interleukin-6 synthesis in osteoblasts: autoregulation by protein kinase C. Cell Signal. 1997; 9: 463-468

24. Vanden Berghe W, Vermeulen L, De Wilde G, De Bosscher K, Boone E, Haegeman G. Signal transduction by tumor necrosis factor and gene regulation of the inflammatory cytokine interleukin-6. Biochem Pharmacol. 2000; 60:1185-1195.

25. Yurchenko V, Zybarth G, O'Connor M, Dai WW, Franchin G, Hao T, Guo H, Hung HC, Toole B, Gallay P, Sherry B, Bukrinsky M. Active site residues of cyclophilin A are crucial for its signaling activity via CD147. J Biol Chem. 2000; 277: 22959-22965.

26. Arora K, Gwinn WM, Bower MA, Watson A, Okwumabua I, MacDonald HR, Bukrinsky MI, Constant SL. Extracellular cyclophilins contribute to the regulation of inflammatory responses. J Immunol. 2005; 175: 517-522.

27. Li L, Tang W, Wu X, Karnak D, Meng X, Thompson R, Hao X, Li Y, Oiao XT, Lin J, Fuchs J, Simeone DM, Chen ZN, Lawrence TS, Xu L. HAb18G/CD147 promotes pSTAT3-mediated pancreatic cancer development via CD44s. Clin Cancer Res. 2013; 19: 6703-6715. 